\title{
Hybrid Large-Eddy/Reynolds-Averaged Simulation of a Supersonic Cavity using VULCAN
}

\author{
Jesse Quinlan \\ University of Virginia \\ 122 Engineer's Way \\ Charlottesville, VA 22904 \\ 757-864-1068 \\ jrq2a@virginia.edu
}

\author{
James McDaniel \\ University of Virginia \\ 122 Engineer's Way \\ Charlottesville, VA 22904 \\ 434-924-6293 \\ jcm@virginia.edu
}

\author{
Robert Baurle \\ NASA Langley Research Center \\ 22 Langley Blvd \\ Hampton, VA 23681 \\ 757-864-9016 \\ robert.a.baurle@nasa.gov
}

\begin{abstract}
Simulations of a supersonic recessed-cavity flow are performed using a hybrid large-eddy/Reynolds-averaged simulation approach utilizing an inflow turbulence recycling procedure and hybridized inviscid flux scheme. Calorically perfect air enters a three-dimensional domain at a free stream Mach number of 2.92. Simulations are performed to assess grid sensitivity of the solution, efficacy of the turbulence recycling, and the effect of the shock sensor used with the hybridized inviscid flux scheme. Analysis of the turbulent boundary layer upstream of the rearward-facing step for each case indicates excellent agreement with theoretical predictions. Mean velocity and pressure results are compared to Reynoldsaveraged simulations and experimental data for each case and indicate good agreement on the finest grid. Simulations are repeated on a coarsened grid, and results indicate strong grid density sensitivity. Simulations are performed with and without inflow turbulence recycling on the coarse grid to isolate the effect of the recycling procedure, which is demonstrably critical to capturing the relevant shear layer dynamics. Shock sensor formulations of Ducros and Larsson are found to predict mean flow statistics equally well.
\end{abstract}

\section{TABLE OF CONTENTS}

1. INTRODUCTION .1

2. PHYSICAL FLOW ..........................................2

3. NUMERICAL FORMULATION ...........................2

4. CoMPUTATIONAL EXECUTION............................3

5. RESULTS.................................................................4

Experimental Comparison ............................... 4

Grid Sensitivity ............................................. 7

Inflow Recycling ............................................. 7

Shock Sensor .................................................. 8

6. CONCLUSIONS ......................................................8

REFERENCES........................................................9

ACKNOWLEDGEMENTS....................................10

\section{INTRODUCTION}

In order to address some of the fundamental limitations of Reynolds-averaged simulation (RAS) approaches while also exploiting the strengths of large-eddy simulation (LES) approaches, researchers frequently use a blended LES/RAS approach. This is especially true for high Reynolds number supersonic wall-bounded flows, which exhibit inherent unsteadiness, turbulent shock-boundary layer interactions, and other complex flow phenomena. Whereas the RAS equations require modeling of turbulence at all length scales, the LES equations only require modeling of turbulent motions at length scales below a characteristic filter width; the largest turbulent motions are resolved naturally through the solution of the filtered governing equations. Since these larger turbulent structures are responsible for the majority of mass, momentum, and energy transport, LES simulations are well-suited for high Reynolds number flows. Unfortunately, the grid densities required for LES simulations of wall-bounded flows often render the approach computationally infeasible for problems of practical importance.

In this study, a hybrid LES/RAS approach is used in conjunction with a turbulence recycling procedure to simulate a supersonic flow over a rearward facing step and subsequent reattachment along an inclined wall [1-3]. The recycling process sustains coherent turbulent structure within the inflow boundary layer without having to simulate its development. Simulation results are compared to experimental mean flow data within the approach turbulent boundary layer, through the shear layer, and along the inclined wall. Assessments of the LES/RAS simulations are made using steady-state RAS results and experimental data.

The simulations are designed to assess several recent developments to the Viscous Upwind aLgorithm for Complex flow ANalysis (VULCAN) computational fluid dynamics (CFD) code, including: reduced-dissipation numerics, a hybridized inviscid flux scheme, and inflow turbulence recycling $[4,5]$. Specifically, the current study: assesses the applicability of the hybrid LES/RAS method for cavity flows, isolates the effect of inflow turbulence recycling on the flowfield statistics, determines any grid sensitivity, and appraises the choice of shock sensor for use with the hybridized inviscid flux scheme. These objectives are achieved by performing seven simulations, which are parameterized by solution method, inflow treatment, grid size, and shock sensor and are summarized in Table 1. Hereafter, each simulation will be referenced using the case ID stated in Table 1. For example, references will be made using "case R35D", rather than "case one", where R, 35, and D indicate recycling, 35 million grid cells, and Ducros sensor, respectively.

In the following section, the physical flow and wind-tunnel experiment are described. In section three, the numerical formulation is presented. After describing the grids and computational execution in section four, the results and accompanying discussion are presented in section five. 
Conclusions and summarizing comments are included in the final section.

Table 1-Simulation cases parameterized by inflow treatment, grid size, and shock sensor.

\begin{tabular}{c|c|c|c|c} 
Case ID & $\begin{array}{c}\text { Solution } \\
\text { Method }\end{array}$ & $\begin{array}{c}\text { Inflow } \\
\text { Treatment }\end{array}$ & $\begin{array}{c}\text { Grid } \\
\text { Cells }\end{array}$ & $\begin{array}{c}\text { Shock } \\
\text { Sensor }\end{array}$ \\
\hline R35D & LES/RAS & Recycling & $35 \mathrm{e} 6$ & Ducros \\
\hline R35L & LES/RAS & Recycling & $35 \mathrm{e} 6$ & Larsson \\
\hline R8D & LES/RAS & Recycling & $8.8 \mathrm{e} 6$ & Ducros \\
\hline R8DW & LES/RAS & Recycling & $35 \mathrm{e} 6$ & Ducros \\
\hline R8L & LES/RAS & Recycling & $8.8 \mathrm{e} 6$ & Larsson \\
\hline NR8D & LES/RAS & $\begin{array}{c}\text { No } \\
\text { Recycling }\end{array}$ & $8.8 \mathrm{e} 6$ & Ducros \\
\hline NR8RAS & RAS & $\begin{array}{c}\text { No } \\
\text { Recycling }\end{array}$ & $8.8 \mathrm{e} 6$ & None
\end{tabular}

\section{Physical Flow}

The flow chosen for the current investigation was first studied by Settles, et. al. in the early 1980s [1-3]. The original experiment was performed in the Princeton University $20 \mathrm{~cm}$ by $20 \mathrm{~cm}$ High Reynolds Number Supersonic Wind Tunnel and consisted of Mach 2.92 air entering the tunnel test section at a stagnation temperature of $258 \mathrm{~K}$, a stagnation pressure of $0.69 \mathrm{MPa}$, and a free stream unit Reynolds number of $6.7 \times 10^{7} / \mathrm{m}$. The test article, presented in Fig. 1, was designed such that a turbulent boundary layer developed along a flat plate before separating over a sharp corner. The free shear layer formed over the subsequent cavity and reattached along a wall of length $18 \mathrm{~cm}$ inclined at $20^{\circ}$. The position of the inclined wall was adjusted before taking measurements until no change in pressure or flow direction occurred due to the separating boundary layer, and the experimenters observed that the shear layer exhibited significant sensitivity to the inclined wall positioning. The cavity depth was $2.54 \mathrm{~cm}$, and the cavity assembly was unaffected by the tunnel sidewall boundary layers due to its positioning away from the walls. In order to promote two-dimensionality, aerodynamic fences lined the edges of the inclined wall. Mean flow measurements were made using a traversing set of pitot and static pressure probes, and these measurements, in conjunction with an assumption of constant total temperature, enabled the researchers to calculate Mach number, velocity, density, and other properties. A spark shadowgraph enabled observation of major flow features (not shown in this paper).

The original experiment was designed to illustrate the complex fluid phenomena comprising the reattachment of a free shear layer in a supersonic flow. The effect of turbulence on the flowfield is prominent, as documented in the original review of the experiment and in several subsequent numerical investigations [2,3]. Major flow features observed in the experiment are illustrated in Fig. 2.
After the approach boundary layer detaches at the step corner, a free shear layer forms over the cavity. As the shear layer reattaches at the inclined wall, fluid is entrained into the cavity, thereby forming a recirculation zone. The inherent unsteadiness of the shear layer alters the location of reattachment and subsequently affects the fluid entrainment. Together, the shear layer unsteadiness, altered attachment point, and recirculation zone form a feedback loop that give rise to a low-frequency flapping motion of the shear layer. Furthermore, as the flow reattaches, an oblique compression shock forms off the inclined wall.

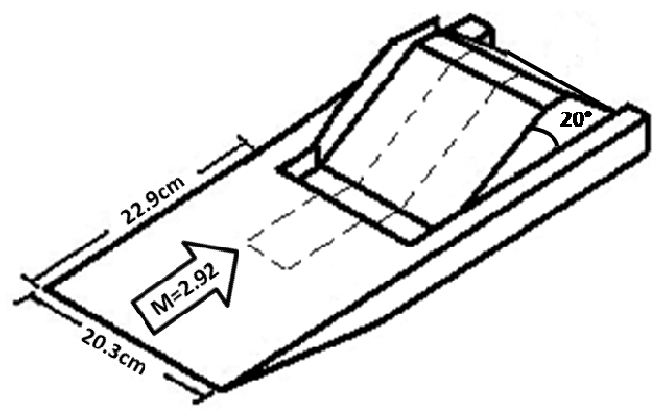

Figure 1-Experimental setup with one aerodynamic fence removed and the current computational domain footprint signified by dashed lines.

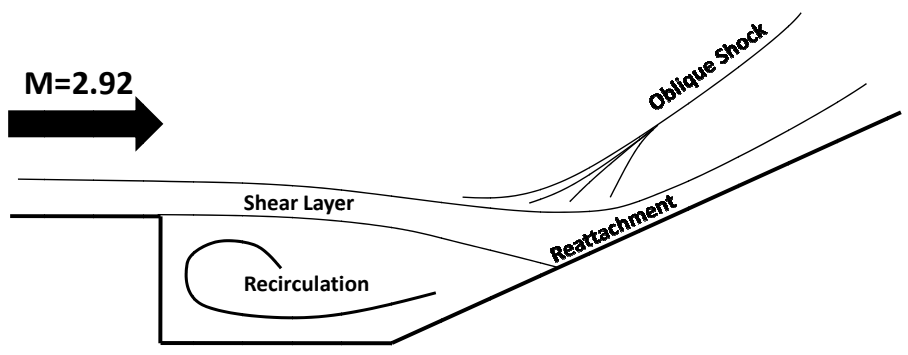

Figure 2-Centerline profile of the experimental apparatus with major flow features highlighted.

\section{NUMERICAL FORMULATION}

All simulations are performed using the VULCAN CFD code, which solves the Navier-Stokes equations using a cellcentered finite-volume approach. Hybridization of the LES and RAS equations is achieved through the blending of the respective closure models. Accordingly, the user has significant flexibility in choosing both the RAS turbulence model and the LES subgrid-scale (SGS) model. Development of the hybrid LES/RAS approach is provided by Baurle, et. al. [6], and details of the current implementation are described by Baurle, et. al. [7].

For each of the LES/RAS simulations, the Menter-BSL turbulence model is used to close the RAS equations [8]. This model requires the solution of two additional equations-a transport equation for turbulent kinetic energy and a transport equation for turbulence frequency. To model the SGS turbulence for use in LES regions, the one- 
equation model of Yoshizawa and Horiuti is used and requires the solution of a transport equation for the SGS turbulence kinetic energy (TKE) [9].

A spatial- and flow-dependent blending function is used to blend the LES and RAS models throughout the computational domain. As designed, the current blending function transitions the RAS formulation to the LES formulation in the outer region of the boundary layer, near the upper edge of the log layer. As previous studies have indicated [10], targeting the transition for this region at the edge of the log layer within the turbulent boundary layer yields the most resolved turbulence without adversely affecting the universality of the boundary layer. The blending function used in the current study is presented by Edwards, et. al. [11].

In order to reduce numerical dissipation in smooth flow regions, while maintaining the dissipation necessary to capture discontinuities elsewhere, a hybridized flux scheme is used to construct interface inviscid fluxes. This hybridization is implemented as the linear combination of a low-dissipation, symmetrically-reconstructed inviscid flux and a conventional upwind-biased flux. The low-dissipation flux formulation uses left and right states constructed to higher-order accuracy at the cell interfaces using a fourpoint symmetric interpolation on the primitive variables [12]. A dissipative flux is then computed by first using the piecewise-parabolic method of Collela and Woodward to construct primitive variables at either side of a cell interface and subsequently using the Harten, Lax, van Leer, and Contact (HLLC) scheme to compute the dissipative flux in conjunction with a minmod-type limiting procedure [10,1315]. The final hybridized inviscid interface flux is computed by blending the low-dissipative and dissipative fluxes using a shock sensor weighting parameter. The two shock sensor formulations of Ducros [16] and Larsson [17] are used in this study. Each of the shock sensors are designed to dynamically adjust the inviscid flux calculation. In regions of viscous, smooth flow, the shock sensor emphasizes the low-dissipation formulation more heavily, whereas in regions of large inviscid gradients, the dissipative flux formulation is more heavily weighted. The lower bound for each of these sensors was modified to provide a small level of background dissipation for the current work. Rather than allowing the sensor to range from 0.0 to 1.0 , each shock sensor is bounded by 0.2 and 1.0 , thereby preventing numerical instabilities arising within the shear layer reattachment region. A detailed development of this hybridized method is presented by White, et. al. [5].

In order to sustain coherent structure within the turbulent inflow boundary layer, turbulent fluctuations and relevant turbulence properties are recycled from a plane one cavitydepth upstream of the step. Fluctuations are extracted from the donor plane by subtracting time- and span-averaged mean flow data from the instantaneous flow data. These fluctuations are scaled according to boundary-layer similarity laws before being superimposed to a mean flow profile. Initial fluctuations are provided by a previouslysimulated fully-resolved (via LES) turbulent boundary layer on a flat plate, which provides the turbulence necessary for efficient recycling without necessitating the natural development of such structures. Details of the recycling procedure are described by Choi, et. al. [10], Xiao, et. al. [18], and Fan, et. al. [19].

\section{Computational Execution}

Simulations are performed on structured three-dimensional fine and coarse grids of 35 and 8.8 million cells, respectively. The coarse grid was constructed by removing every other grid point in each of the streamwise and spanwise directions of the fine grid. The grid generated for this study is composed of four primary blocks, as shown in Fig. 3. The two upper blocks are connected to the lower blocks via non-C0 continuous patches. Similarly, the lower left block is attached to the lower right block by a non-C0 continuous patch where only the spanwise coordinates are mismatched (the downstream spacing is twice that of the upstream spacing). On the fine grid, streamwise-transversespanwise dimensions of the blocks adjacent to the wall directly upstream and downstream of the step are $525 \times 121 \times 301$ and $453 \times 241 \times 151$, respectively; dimensions of the upper left and right blocks are $29 \times 45 \times 15$ and $153 \times 45 \times 15$, respectively. The patches connecting the lower and upper blocks are positioned far enough from the domain of interest to ensure that resolved boundary layer and free shear layer eddies are not convected into them.

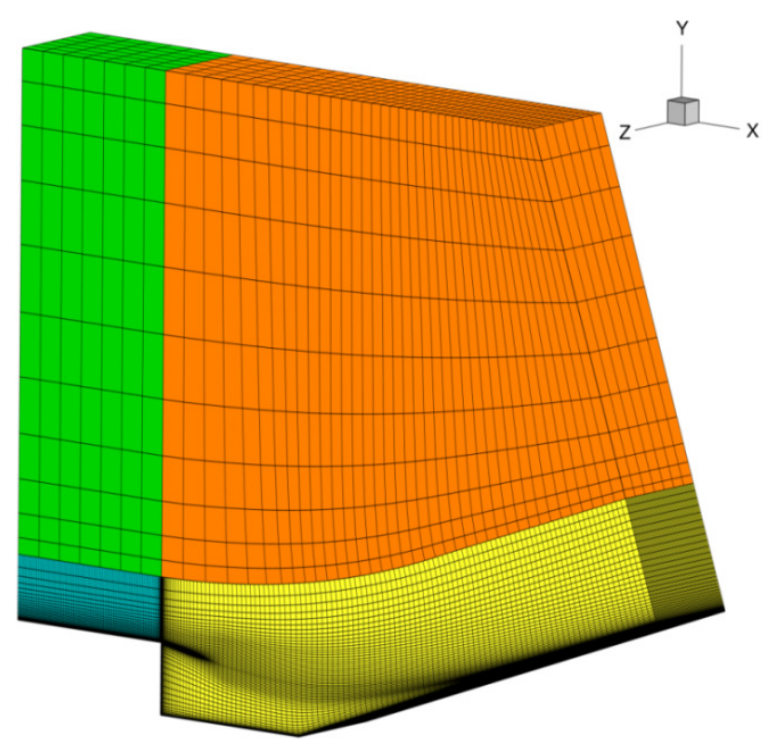

Figure 3-Three-dimensional domain coarsened twice in the streamwise and transverse directions and once in the spanwise dimension for visual clarity.

The physical dimensions of the computational domain are $28.6 \mathrm{~cm}, 22.5 \mathrm{~cm}$, and $3.81 \mathrm{~cm}$ in each of the streamwise, transverse, and spanwise directions, respectively. The flat plate extended four step heights upstream of the step, which is a factor of two further upstream than in previous studies to allow for recycling of turbulent fluctuations. The grid 
was clustered in the wall-normal direction to provide a nominal $\mathrm{y}+$ of $1.0[5,20]$. The streamwise and spanwise grid spacing was set to $1 / 20$ th of the boundary layer thickness. Downstream of the step, the grid clustering required by the approach flow boundary layer was relaxed toward a more isotropic grid for the resulting shear layer. The average streamwise, transverse, and spanwise spacing in this region was $2.0 \%, 0.2 \%$, and $1.0 \%$, respectively, of the cavity depth $(2.54 \mathrm{~cm})$. The cell aspect ratio for the LES regions was no greater than approximately 20.0 . The wide grid used for case R8DW extended 4.5 cavity depths in the spanwise direction and maintained the same grid spacing and clustering as the coarse grid. The width of the computational domain was increased by a factor of three to investigate potential correlations between two-point statistics that may be present in the original domain size. The three-dimensional fine and coarse grids were split into 1980 sub-blocks to allow runtime parallelization using Message Passing Interface (MPI) on NASA Advanced Supercomputing resources. Each simulation performed on the fine grid required approximately 360 hours of wall clock time on 512 processors. The same simulations performed on the coarse grid required about 90 hours of wall clock time on 512 processors.

Inflow profiles and flowfield initializations for the LES/RAS cases were obtained using the NR8RAS case, in which the Menter k- $\omega$ turbulence model was used with a compressibility correction [8,21]. Further, the free stream flow was allowed to develop until the momentum thickness one cavity-depth upstream of the step matched experimental measurements. A significant spread in solutions was obtained using various turbulence models for the preliminary RAS simulation, and the RAS results used in this study represent those in best agreement with experimental data. Nominal inflow conditions included: static pressure of $21.2 \mathrm{kPa}$, Mach number of 2.92 , and temperature of $92.4 \mathrm{~K}$. For all LES/RAS cases, periodic boundary conditions were used in the homogeneousspanwise direction. All walls were assumed adiabatic and no slip, except for the vertical wall during coarse grid runs, where wall functions were applied due to excessively-high $\mathrm{y}+$ values observed there during runtime. For all runs, an extrapolation condition was applied at the exit plane, and a characteristic condition was enforced at the far-field plane.

The governing equations were integrated using a dual-timestepping approach, in which the diagonalized approximate factorization (DAF) method is used for integration in pseudo time with a maximum Courant-Friedrichs-Lewy (CFL) number of 100.0, and a second-order three-point backwards finite difference approximation was used for integration in physical time [22,23]. Convergence was defined as achieving a residual reduction of 2.5 orders-ofmagnitude or as reaching a limiting number of 15 sub- iterations. The physical time step was set to $0.1 \mu$ s for each LES/RAS simulation. Following initialization, the flowfield was evolved for a minimum of 25 characteristic flowthrough times before taking statistics. A characteristic flowthrough time was defined as the time required for a particle in the free stream to travel from the corner of the step to the point of reattachment of the free shear layer along the inclined wall-approximately $0.2 \mathrm{~ms}$. Statistical stationarity was confirmed by comparing independent sets of statistics gathered at intervals of five flow-through times. After 25 flow-through times, the flowfield was statistically stationary, and statistics were gathered for a final five flowthrough times at a rate of $0.5 \mu \mathrm{s}$. These statistics were subsequently averaged spatially in the homogeneous spanwise direction. The RAS turbulence model constants were set according to Menter [8], and SGS turbulence model constants were set as suggested by Yoshizawa and Horiuti [9].

\section{RESULTS}

The simulations reproduced the major flow phenomena observed in the experiment, as seen in Fig. 4; the supersonic flow upstream of the cavity detaches at the step corner, thereby creating a free shear layer over the cavity. This free shear layer attaches along the inclined wall and interacts with an oblique shock front standing off the inclined wall. Fluid is entrained into the cavity near the location of shear layer attachment, and this fluid creates a recirculation zone which drives an unsteady low-frequency motion of the free shear layer. Note that in Fig. 4, annotations are included that indicate major flow features and identify each image's relative location in time, which is given as the value of elapsed time, $\Delta \mathrm{t}$, divided by the characteristic flow-through time, $\Delta \tau$. In the following subsections, results are presented and organized according to study objective, namely: assessment of solution accuracy via comparison to experiment for the baseline case (R35D), comparison of results on the narrow (R8D) and wide (R8DW) grids, assessment of grid sensitivity by comparing fine grid solutions (R35D and R35L) to those on the coarse grid (R8D and R8L), determination of inflow turbulence recycling effects by comparing cases with (R8D) and without recycling (NR8D), and determination of shock sensor effects by comparing cases performed with the Ducros switch (R35D and R8D) to those performed with the Larsson switch (R35L and R8L).

\section{Experimental Comparison}

Before comparing results to experimental data, the approach boundary layer was confirmed to have developed properly. This confirmation was necessary to ensure that the recycling procedure did not adversely affect the inflow boundary layer statistics. For both the fine grid and coarse grid cases, boundary layer velocity profiles at several planes upstream 


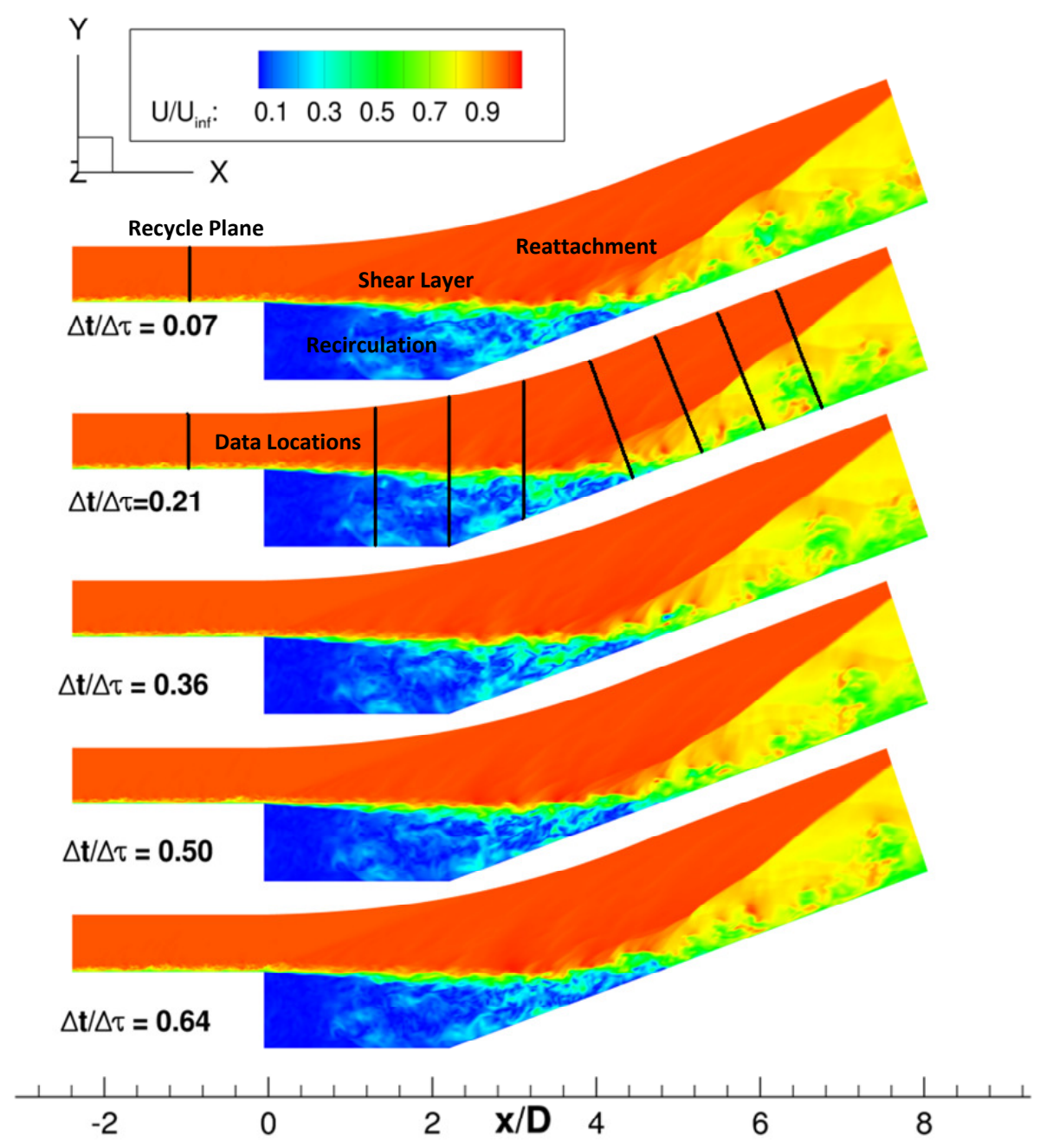

Figure 4-Instantaneous snapshots of velocity magnitude at the spanwise centerline plane for case R35D with the recycle plane, major flow features, and data extraction locations marked.

of the step were compared to theoretical predictions based on Cole's Law-of-the-Wall formulation and the original RAS solutions under the van Driest transformation described by Choi, et. al. [10]. These three planes included the inflow plane, the donor recycling plane, and a plane at the step corner. For each case and at each plane, the averaged LES/RAS results agreed well with both the theoretical log-layer predictions and with RAS data. The comparisons for cases R35D and R8D at the recycling plane are included in Fig. 5 to demonstrate the level of agreement. In each case and at each plane, the LES/RAS simulations reproduced the slope and location of the log-layer well, and the blending function anchored the mean LES/RAS transition point near the upper edge of the log-layer. Thus, the LES/RAS approach and recycling procedure provide proper development of the boundary layer upstream of the step.

Mean velocity and static pressure at several planes are compared with experimental data in Fig. 6 for each case, though details of the baseline case, R35D, are highlighted in this section. The results of cases R35L, R8L, and R8DW are omitted from Fig. 6 due to their superb agreement with cases $\mathrm{R} 35 \mathrm{D}$, R8D, and $\mathrm{R} 8 \mathrm{D}$, respectively, and will be discussed later. Note that in Fig. 6, an $*$ denotes a value normal to the wall, $\infty$ indicates a free stream condition, $\delta$ is the approach boundary layer thickness, and all spatial dimensions are non-dimensionalized by the cavity depth, $D$. The results of case R35D agree well with the experimental data, except within the cavity region. Static pressure along the inclined wall and at the chosen planes normal to the inclined wall agree well with experiment and mark an increase in accuracy, as compared to case NR8RAS. The velocity data at planes normal to the inclined wall also mark an increase in accuracy. The improved agreement in static pressure and velocity data along the inclined wall is no surprise, as it is known that RAS models tend to severely over-predict the boundary layer recovery rate when free shear flows reattach to solid surfaces. However, while case R35D more accurately predicts the shear layer spreading rate, it is apparent the shear layer is deflected toward the cavity floor. Forming a "best-fit" line anchored at the step corner that follows the shear layer formed in the R35D case results in a deflection angle of $2.85^{\circ}$. 


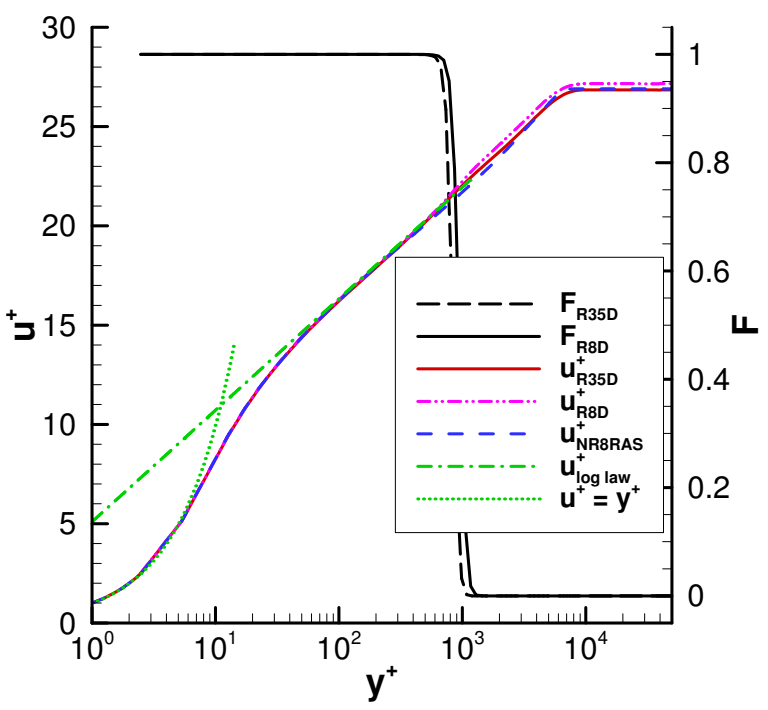

Figure 5-Velocity and blending function in the approach boundary layer for cases R35D and R8D at the recycling plane.
Case R8DW was performed to determine whether slowmoving, large-scale motions within the cavity were artificially limited on the baseline computational domain. The results of this case confirmed that no such artificial forcing occurred on the original narrow coarse grid. Therefore, the authors posit that the experimental data may exhibit effects of the tunnel sidewalls and aerodynamic fences, which were not modeled in the current study; previous efforts have shown that three-dimensional effects play a significant role in flow separation even when extensive steps are taken in the experiments to ensure some level of two-dimensionality [24]. In order to clearly illustrate shear layer spreading rate comparisons, each of the cavity velocity profiles are shifted vertically in Fig. 7 until the point of inflection is approximately aligned with that of the experimental data. As expected, fine grid cases predict the spreading rate well, and coarse grid cases display weaker agreement. Case R35D indicates significant improvement as compared to the other cases. It should be noted that case NR8D is in less agreement than R8D, which indicates an effect of recycled turbulent structure on shear layer development and will be discussed later.

$x / D=-1.0 \quad x / D=1.5 \quad x / D=2.5 \quad x / D=3.5$
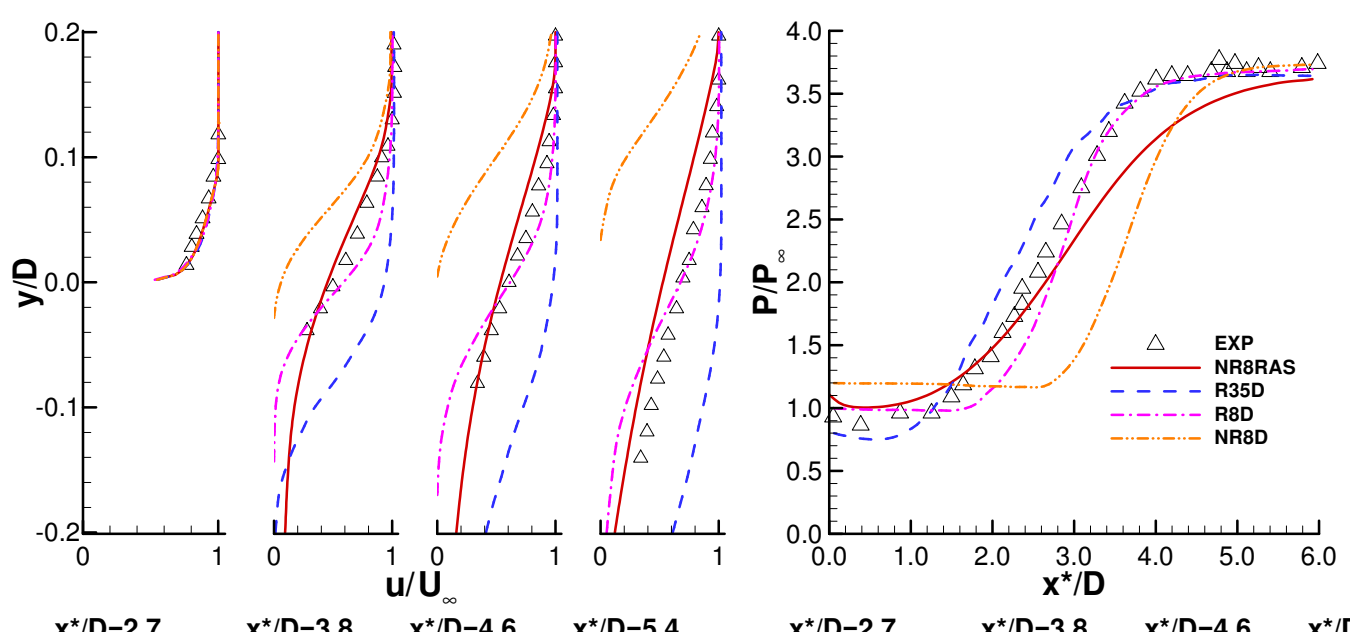

$\mathrm{x}^{\star} / \mathrm{D}=3.8 \quad \mathrm{x}^{\star} / \mathrm{D}=4.6 \quad \mathrm{x}^{\star} / \mathrm{D}=5.4$

$x^{\star} / D=2.7$
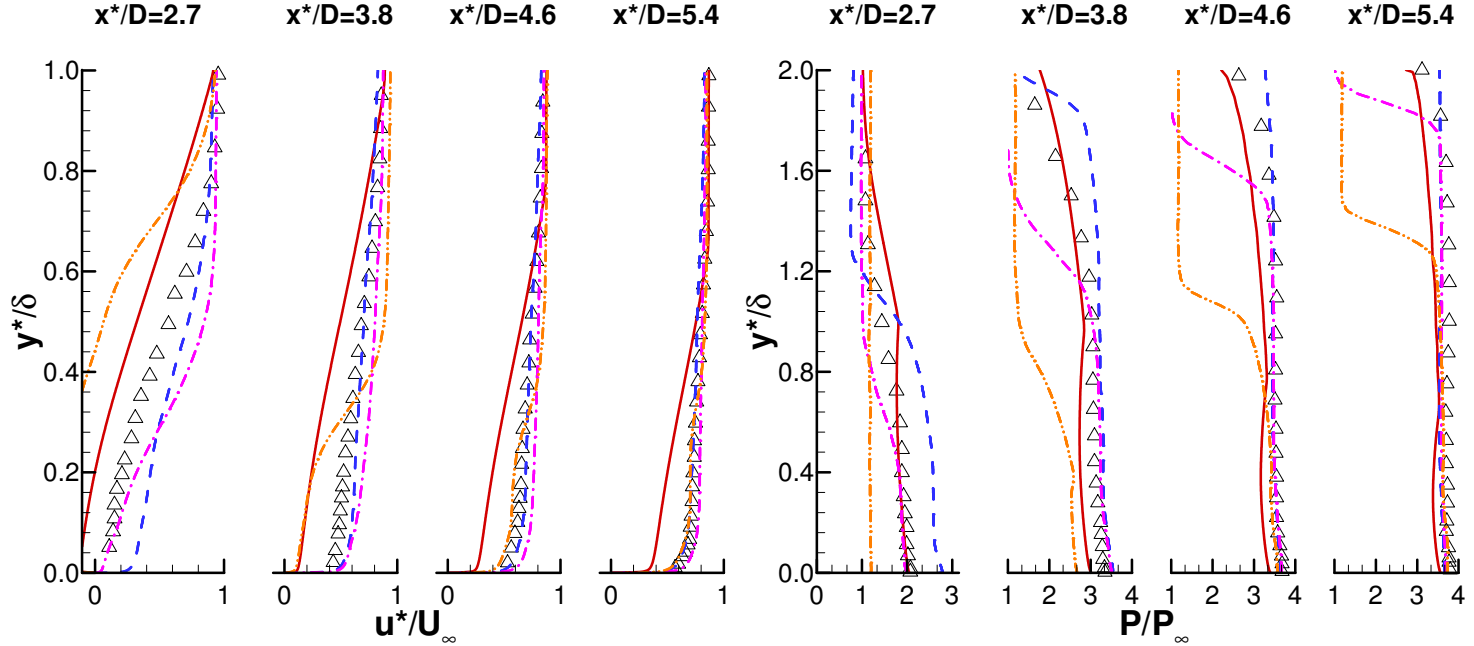

Figure 6-Velocity and pressure for cases R35D (blue dashed line), R8D (magenta dash-dot line), NR8D (orange dashdot-dot line), and NR8RAS (solid red line) to experiment (open triangles) at locations highlighted in Fig. 4. 


\section{Grid Sensitivity}

Grid sensitivity was assessed by comparing results of cases R35D and R8D. Case R8D utilized a once-coarsened version of case R35D's grid in the streamwise and spanwise directions; coarsening the transverse direction would have yielded unacceptably-large values of $y+$ for integrate-to-thewall solutions. Based on these two cases, the grid sensitivity of the solution is significant. The coarsened grid fails to resolve adequate levels of turbulence, as compared to the fine grid, which is illustrated in Fig. 8, where instantaneous Mach number contours at the spanwise center-line are presented. Note that while the turbulent fluctuations in the approach flow boundary layer are wellresolved for both cases, these fluctuations and corresponding turbulent motions are resolved to a lesser degree moving downstream of the step on the coarse grid.

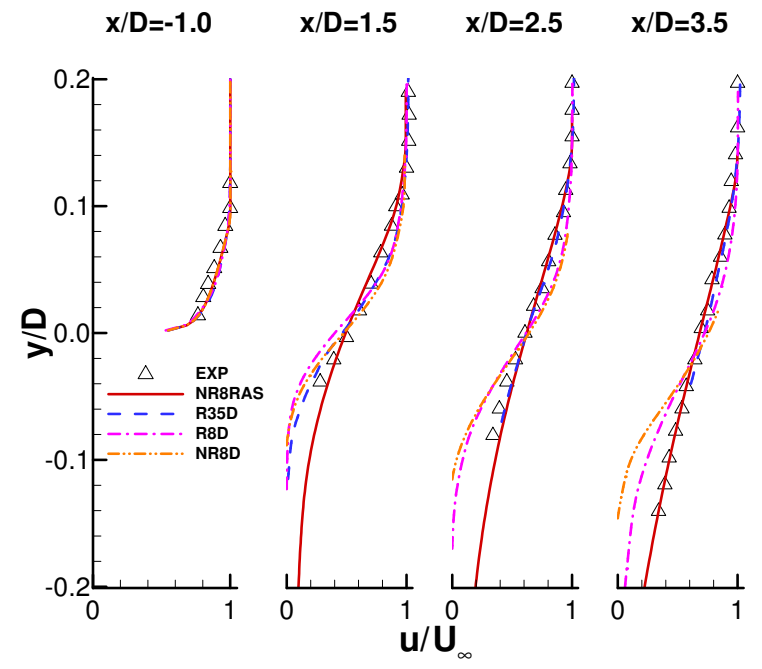

Figure 7-Velocity profiles translated vertically to demonstrate spreading rate agreement for cases R35D (blue dashed line), R8D (magenta dash-dot line), NR8D (orange dash-dot-dot line), and NR8RAS (red solid line) compared to experiment (open triangles).
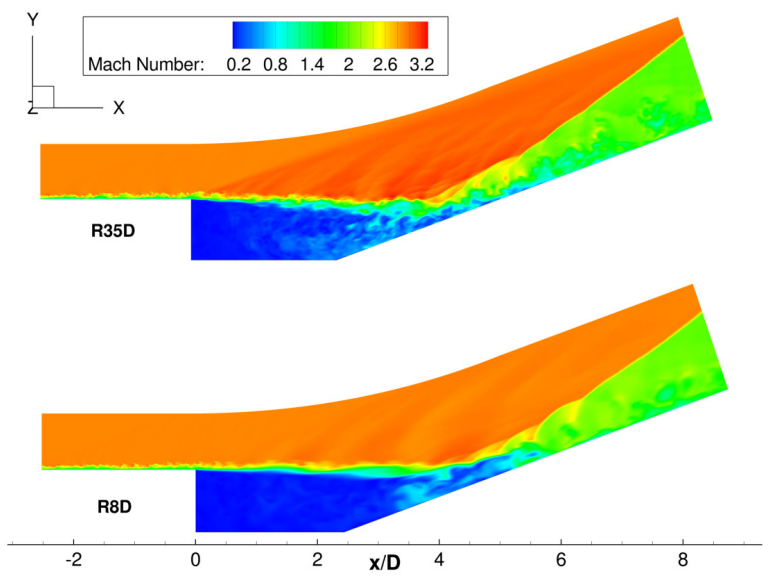

Figure 8-Instantaneous Mach number snapshots for cases $\mathrm{R35D}$ (top) and $\mathrm{R} 8 \mathrm{D}$ (bottom) at the spanwise centerline plane.
This observation is quantified by examining resolved TKE and SGS TKE contributions downstream of the step, which are plotted in Fig. 9 at several locations through the shear layer. The percentage of resolved turbulence is calculated according to Eq. (1), where $\mathrm{TKE}_{\mathrm{res}}$ and $\mathrm{TKE}_{\mathrm{sgs}}$ are the resolved SGS TKE, respectively.

$$
\% T K E_{\text {res }}=\frac{T K E_{\text {res }}}{T K E_{S G S}+T K E_{\text {res }}} \times 100
$$

Peak-to-peak values from Fig. 9 are used with Eq. (1). Whereas in case $\mathrm{R} 35 \mathrm{D}$, the turbulence is $71 \%, 80 \%$, and $85 \%$ resolved at each plane, respectively, the turbulence is only $30 \%, 36 \%$, and $50 \%$ resolved at the same planes for case R8D. Typically, the desired level of resolved turbulence for LES of high Reynolds number flows is in the $80-90 \%$ range [25-27]. In case $\mathrm{R} 8 \mathrm{D}$, the dominance of $\mathrm{TKE}_{\mathrm{sgs}}$ leads to more reliance on the SGS model, which in this case yields a larger disagreement with the experimental data.
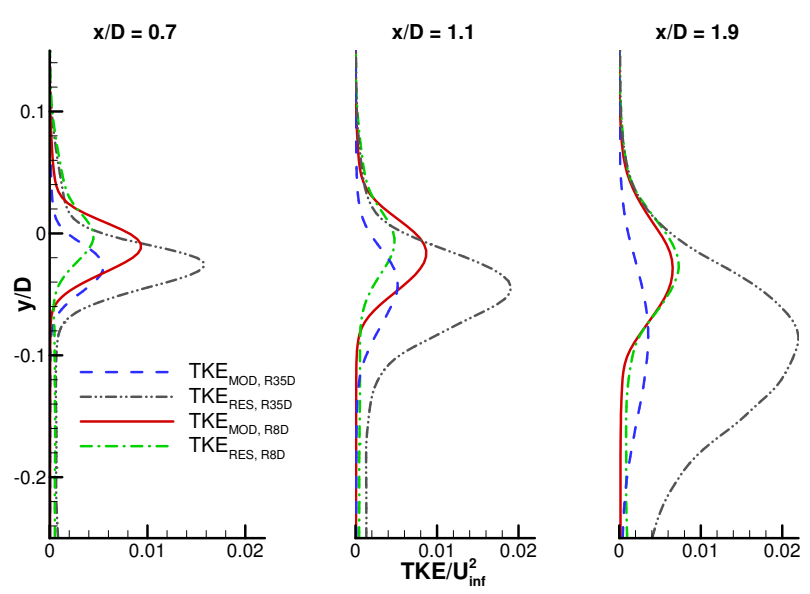

Figure 9-TKE profiles for cases R35D and R8D. The blue dashed line and red solid line are the SGS TKE for cases R35D and R8D, respectively, and the gray dashdot-dot line and green dash-dot line are the resolved TKE for cases $R 35 D$ and $R 8 D$, respectively.

\section{Inflow Recycling}

The effect of inflow turbulence recycling on the mean flowfield statistics was isolated by rerunning case R8D without recycling. The flow upstream of the step was simulated using pure RAS, since without the sustained coherent turbulent structure within the approach boundary layer, the boundary layer was at risk of partially relaminarizing. As can be seen in Figs. 6 and 7, the results of case NR8D are significantly different from those of case R8D; the shear layer is significantly higher, the spreading rate is lower, the static pressure within the cavity is higher, and the shear layer reattachment point is further downstream. From the pressure data, it is evident that the oblique shock makes a shallower angle with the inclined wall as compared to case R8D. Explanation of such a discrepancy requires consideration of several codependent fluid mechanical phenomena, including the transport of 
mass through the shear layer and the reattachment point's impact on mass entrainment into the cavity. Turbulent transport through the shear layer is illustrated in Fig. 10 by lineplots of the Reynolds shear stress component, $\overline{u^{\prime \prime} v^{\prime \prime}}$. Through the shear layer, significantly less mass transfer occurs for case NR8D as compared to cases NR8RAS, R8D, and $\mathrm{R} 35 \mathrm{D}$, thereby driving the static pressure within the cavity higher. As the shear layer moves vertically, the reattachment point moves further downstream, thereby reducing the incident angle of the shear layer with the inclined wall. As this angle lessens, the amount of fluid being entrained into the cavity reduces further. Contrary to earlier examinations of this flow, the current results demonstrate the criticality of sustaining coherent turbulence within the approach boundary layer when employing the most recent developments to VULCAN for such a flow $[5,28]$.
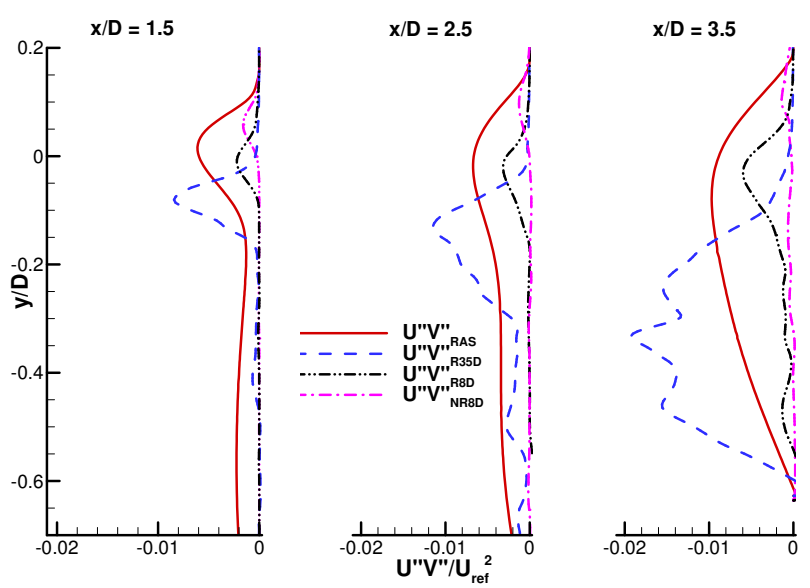

Figure 10-Reynolds shear stress component for cases NR8RAS (red solid line), R35D (blue dashed line), R8D (black dash-dot-dot line), and NR8D (magenta dash-dot line).

\section{Shock Sensor}

The effect of shock sensor choice is assessed by comparing cases R35D and R35L and cases R8D and R8L. The Ducros sensor was considered the baseline for this study. Both shock sensors are designed to compare the magnitude of the divergence and curl of velocity; however, the Larsson sensor includes logic that toggles the interface flux approximation to either fully-upwind or fully-symmetric, versus the Ducros sensor's blending of the two. The result of this difference is illustrated in Fig. 11, where an instantaneous snapshot of the shock sensor is presented for cases R8D and R8L. Note how the Larsson sensor tends to confine the upwind flux formulation to regions near the oblique shock. The Ducros switch uses the centrallydifferenced flux formulation through most of the cavity region and behind the oblique shock. However, the Ducros switch employs the upwind flux formulation above the shear layer, since by design, any small fluctuation in otherwise smooth, inviscid flow triggers such switch behavior. Results of cases R35L and R8L are statistically equivalent to those of R35D and R8D, respectively. For such reason, the results are omitted from Fig. 6, and therefore, the mean flow statistics are independent of whether the Ducros or Larsson shock sensor is used for the current simulations.
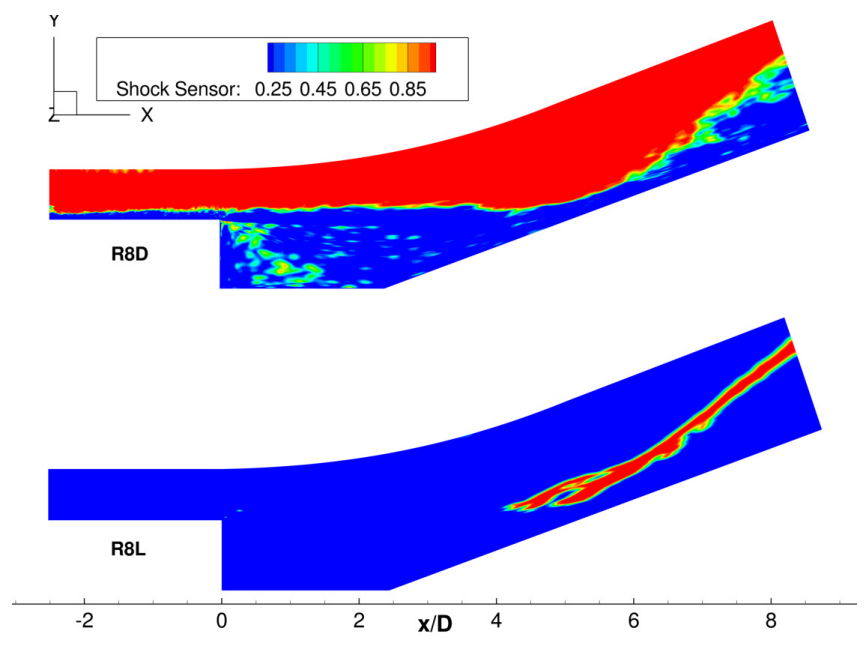

Figure 11-Instantaneous shock sensor contours for cases R8D (top) and R8L (bottom) at the spanwise centerline plane.

\section{Conclusions}

Simulations of a supersonic cavity flow exhibiting a reattaching free shear layer utilizing recent developments to the VULCAN CFD code were conducted in order to: assess the applicability of LES/RAS, isolate the effect of inflow turbulence recycling, determine any sensitivity of the solution to grid density, and evaluate the effect of shock sensor choice for use with a hybridized flux scheme. Two grids were used - a fine grid consisting of 35 million cells and a coarsened version of the same grid constructed of 8.8 million cells. A baseline run was performed on the fine grid using the Ducros sensor. Two cases were performed on the coarsened grid with the Ducros switch toggling the inflow turbulence recycling on and off. The cases performed with recycling were repeated using the Larsson switch to isolate the effect of shock sensor choice. Results utilizing the fine grid compared quite well with experimental data outside of the cavity region. Through the cavity region, velocity profiles indicated excellent shear layer spreading rate agreement but disagreement in vertical location of the shear layer. This discrepancy may be the result of threedimensional sidewall effects not considered in the current study. Even though measures were taken during the experiment to promote two-dimensionality, such as aerodynamic fences, previous studies have indicated that such approaches likely do not completely alleviate the effects of three-dimensionality [24]. Based on the comparisons of cases $\mathrm{R} 35 \mathrm{D}$ and $\mathrm{R} 8 \mathrm{D}$, the solution is strongly-sensitive to grid density. Grid convergence was not empirically proven based on mean statistics; however, simulations performed on the fine grid proved to resolve a reasonable fraction of the turbulence, whereas the coarse grid simulations exhibited dominance of the SGS modeled 
turbulence. Cases R8D and NR8D suggest inflow turbulence recycling plays a pivotal role in proper shear layer development. Without recycling, significantly less mass is exchanged through the shear layer, allowing higher static pressures to build within the cavity. Subsequently, as the shear layer lifts, less fluid is entrained into the cavity, and the reattachment point moves further downstream. The oblique shock makes a shallower angle with the inclined wall, thereby causing severe disagreement in velocity and pressure data as compared to experiment. Finally, the choice of shock sensor was found to have a negligible effect on the mean flow statistics. The sensors of Ducros and Larsson were considered, and the results illustrate the relative insensitivity to the detailed functional form chosen for the shock sensor. Future work will address the threedimensionality concerns raised in the current study.

\section{REFERENCES}

[1] Settles, G.S., Baca, B.K., Williams, D.R., and Bogdanoff, S.M., "A study of reattachment of a free shear layer in compressible turbulent flow," AIAA Paper 80-1408, July 1980 .

[2] Horstman, C.C, Settles, G.S., Bogdanoff, S.M., and Williams, D.R., "A reattaching free shear layer in compressible turbulent flow-A comparison of numerical and experimental results," AIAA Paper 81-0333, Jan. 1981.

[3] Settles, G.S., Williams, D.R., Baca, B.K., and Bogdanoff, S.M., "Reattachment of a compressible turbulent free shear layer," AIAA J., Vol. 20(1), 1982, pp. 60-68.

[4] "Viscous Upwind Algorithm for Complex Flow Analysis (VULCAN): User Manual," Online, 2010.

[5] White, J.A., Baurle, R.A., Fisher, T.C., Quinlan, J.R., and Black, W.S., "Low-dissipation advection schemes designed for large eddy simulations of hypersonic propulsion systems," AIAA Paper 2012-4263, July 2012.

[6] Baurle, R.A., Tam, C.J., Edwards, J.R., and Hassan, H.A., "A hybrid simulation approach for cavity flows: blending, algorithm, and boundary treatment issues," AIAA J., Vol. 41(8), 2003, pp. 1463-1480.

[7] Baurle, R.A. and Edwards, J.R., "Hybrid Reynoldsaveraged/large-eddy simulations of a coaxial supersonic freejet experiment," AIAA J., Vol. 48(3), 2010, pp. 551571 .

[8] Menter, F.R., "Zonal two equation k- $\omega$ models for aerodynamic flows," AIAA Paper 92-0439, Jan. 1992.
[9] Yoshizawa, A., and Horiuti, K., “A statistically-derived subgrid scale kinetic energy model for large-eddy simulation of turbulent flows," J. Phys. Soc. of Japan, Vol. 54, 1985, pp. 2834.

[10] Choi, J.-I., Edwards, J.R., and Baurle, R.A., "Compressible boundary layer predictions at high Reynolds number using hybrid LES/RANS methods," AIAA Paper 2008-4175, June 2008.

[11] Edwards, J.R., Choi, J.-I., and Boles, J.A., "Hybrid LES/RANS simulation of a mach 5 compression-corner interaction,” AIAA J., Vol. 46(4), 2008, pp. 977-991.

[12] Gieseking, D., Choi, J-I., Edwards, J., Hassan, H., "Simulation of shock/boundary layer interactions using improved LES/RANS models," AIAA Paper 2010-111, Jan. 2010.

[13] Colella, P., and Woodward, P.R., "The piecewise parabolic method (PPM) for gas-dynamical simulations," J. Comp. Phys., Vol. 54, 1984, pp. 174-201.

[14] Batten, P., Clarke, N., Lambert, C., and Causon, D.M., "On the choice of wave speeds for the HLLC Riemann Solver," SIAM J. Sci. Comp., Vol. 18(6), 1997, pp. 15531570 .

[15] Toro, E.F., Spruce, M., and Speares, W., "Restoration of the contact surface in the HLL-Riemann solver," Shock Waves, Vol. 4(1), 1994, pp. 25-34.

[16] Ducros, R., Ferrand, V., Nicoud, F., Weber, C., Darracq, D., Gacherieu, C., and Poinsot, T., "Large-eddy simulation of shock-turbulence interaction," J. Comp. Phys., Vol. 152(2), 1999, pp. 517-549.

[17] Larsson, J., Vicquelin, R., and Bermejo-Moreno, I., "Large-eddy simulations of the HyShot II scramjet," Center for Turbulence Research Annual Research Report Briefs, 2011.

[18] Xiao, X., Edwards, J.R., Hassan, H.A., and Baurle, R.A., "Inflow boundary conditions for hybrid largeeddy/Reynolds-averaged Navier-Stokes simulations," AIAA J., Vol. 41(8), 2003, pp. 1481-1490.

[19] Fan, T.C., Edwards, J.R., Hassan, H.A., and Baurle, R.A., "Hybrid large-eddy/Reynolds-averaged NavierStokes simulations of shock separated flows," J. of Spacecraft and Rockets, Vol. 41(6), 2004, pp. 897-906.

[20] Fan, T.C., Xiao, X., Edwards, J.R., Hassan, H.A., and Baurle, R.A., "Hybrid RANS/LES simulation of a shock wave/boundary layer interaction," AIAA Paper 20020431, Jan. 2002.

[21] Wilcox, D.C., Turbulence Modeling for CFD, 1st ed., DCW Industries, Inc., 1993. 
[22] Rai, M.M., "Navier-Stokes simulations of blade vortex interaction using high-order accurate upwind schemes," AIAA Paper 87-0543, 1987.

[23] Pulliam, T.H., "A diagonal form of an implicit approximate-factorization algorithm," J. Comp. Phys., Vol. 39(2), 1981, pp. 347-363.

[24] Oliver, A.B., Lillard, R.P., Schwing, A.M., Blaisdell, G.A., and Lyrintzis, A.S., "Assessment of turbulent shock-boundary layer interaction computations using the OVERFLOW code," AIAA Paper 2007-0104, Jan. 2007.

[25] Pope, S.B., “Ten questions concerning the large-eddy simulation of turbulent flows," New J. Phys., Vol. 6(1), 2004.

[26] Sagaut, P., Large-eddy simulations of incompressible flows: an introduction, Springer, Berlin, Germany, 2006.

[27] Pope, S.B., Turbulent Flows, Cambridge University Press, New York, NY, 2000.

[28] Quinlan, J.R., Baurle, R.A., and McDaniel, J.C., "Simulation of a wall-bounded flow using a hybrid LES/RAS approach with turbulence recycling," AIAA Paper 2012-3285, June 2012.

\section{Acknowledgements}

This work was supported by a NASA Space Technology Research Fellowship (training grant NNX11AM86H), with additional support provided by the Virginia Space Grant Consortium and by a Virginia Commonwealth Fellowship. The authors are grateful for the helpful discussions with Dr. Tomasz Drozda during the course of this study. Computational resources were provided by the NASA Advanced Supercomputing Division. 